\title{
United States Military Cancer Institute
}

National Cancer Institute

\section{Source}

National Cancer Institute. United States Military Cancer Institute. NCI Thesaurus. Code C79868.

A tri-service program whose goal is to initiate, develop, coordinate and enhance multiinstitutional cancer research, education and care within the military system. 